\title{
Aberrant histone modifications induced by mutant ASXL1 in myeloid neoplasms
}

\author{
Shuhei Asada ${ }^{1} \cdot$ Toshio Kitamura $^{1}$
}

Received: 12 November 2018 / Accepted: 19 November 2018 / Published online: 5 December 2018

(c) The Japanese Society of Hematology 2018

\begin{abstract}
An epigenetic modulator Additional sex combs-like 1 (ASXL1) is recurrently mutated in myeloid neoplasms such as myelodysplastic syndromes (MDS), acute myeloid leukemia (AML) and myeloproliferative neoplasms (MPNs). ASXL1 mutations are also frequently detected in clonal hematopoiesis with indeterminate potential (CHIP), which is the clonal expansion of premalignant hematopoietic cells without any evidence of hematological malignancies. Thus, understanding the roles of ASXL1 in hematopoiesis and myeloid neoplasms is a clinically crucial issue. ASXL1 mutations in hematological neoplasms are typically frameshift or nonsense mutations and occur near the $5^{\prime}$ end of the last exon, thereby the transcripts would escape from nonsense-mediated decay, Indeed, we identified the C-terminally truncated mutant protein of ASXL1 in several cell lines derived from patients with myeloid leukemia. In mouse models, expression of the mutant ASXL1 results in impaired hematopoiesis and promotes development of myeloid neoplasms. In addition, recent findings from biochemical analysis have demonstrated that the mutant ASXL1 protein gains new functions including enhancing catalytic activity of BRCA1-associated protein 1 (BAP1), resulting in reduction of H2AK119ub and aberrant gene expression essential for myeloid transformation. In this review, we will focus on the pivotal roles of the mutant ASXL1 on histone modifications and myeloid transformation.
\end{abstract}

Keywords ASXL1 $\cdot$ Histone modifications $\cdot$ H2AK119ub $\cdot$ BAP1 $\cdot$ Myeloid neoplasms

\section{Introduction}

Recent next-generation sequence studies have uncovered mutational landscape in various types of hematological neoplasms. Additional sex combs-like 1 (ASXL1) is one of the most frequently mutated genes in myeloid malignancies including myelodysplastic syndromes (MDS) [1, 2], acute myeloid leukemia (AML) [3-5] and myeloproliferative neoplasms (MPNs) [6, 7]. ASXL1 mutations are found in about a half of chronic myelomonocytic leukemia (CMML) [8-11]. Of note, ASXL1 mutations are associated with poor prognosis $[1,3-5,8,10]$. In addition, $A S X L 1$ mutations are recurrently found in healthy individuals with clonal

Toshio Kitamura

kitamura@ims.u-tokyo.ac.jp

1 Division of Cellular Therapy, Advanced Clinical Research Center, Division of Stem Cell Signaling, Center for Stem Cell Biology and Regenerative Medicine, Institute of Medical Science, The University of Tokyo, 4-6-1 Shirokanedai, Minato-ku, Tokyo 1088639, Japan hematopoiesis with indeterminate potential (CHIP) as with DNA methyltransferase 3A (DNMT3A) and Ten-eleventranslocation 2 (TET2) [12-14]. CHIP is characterized by clonal expansion with somatic preleukemic mutations in absence of any evidence of hematological malignancies. CHIP is associated with an increased risk of development of hematological neoplasms [12-14]. A recent study demonstrated that CHIP is also an independent risk for coronary heart disease [15].

Therefore, understanding the roles of ASXL1 in hematopoiesis is much important. Recent studies have revealed that knockdown of Asxl1 accelerates N-ras induced myeloid transformation [16]. Asxll knockout mice exhibited impaired hematopoiesis and development of MDS-like disease $[17,18]$. These findings indicated that wildtype ASXL1 plays crucial roles as a tumor suppressor in hematopoiesis and suggested loss-of-function features of ASXL1 mutations. However, most ASXL1 mutations in hematological neoplasms occur near the last exon and its mutations are frameshift or nonsense mutations, thereby its transcripts would escape from nonsense-mediated decay. C-terminally 
truncated form of ASXL1 protein are detectable by Western blotting analysis and mTRAQ-based mass spectrometric analysis [19]. Biological findings and biochemical analysis of the mutant ASXL1 have demonstrated that the mutant ASXL1 accelerates myeloid neoplasms in a dominant-negative and gain-of function manner.

In this review, we will focus on the findings from recent biological studies how the mutant ASXL1 impairs normal hematopoiesis and promotes myeloid neoplasms. We will also introduce the potential therapeutic strategy of myeloid neoplasms harboring ASXL1 mutations.

\section{The structure of ASXL family proteins}

Mammalian three ASXL family genes (ASXL1, ASXL2 and ASXL3) are the mammalian homologs of Drosophila Additional sex combs (Asx) [20]. Drosophila Asx was originally identified as an enhancer of trithorax and polycomb (ETP) and required for both gene activation and repression [21, 22]. Drosophila Asx forms polycomb repressive deubiquitinase complex (PR-DUB) with a histone deubiquitinase Calypso [23]. The mammalian homolog of Drosophila Calypso is BRCA-1 associated protein 1 (BAP1). Mammalian ASXL1 and BAP1 also form a mammalian PR-DUB complex, which removes ubiquitination of $\mathrm{H} 2 \mathrm{~A}$ at lysine 119 (H2AK119ub). H2AK119ub modification is associated with a repressive chromatin status and catalyzed by an E3 ligase RING1A/
RING1B, which is a main component of Polycomb repressive complex 1(PRC1) [24, 25].

Mammalian ASXL1 and ASXL2 are ubiquitously expressed in a variety of tissues, whereas the expression of ASXL3 is low overall and restricted to lymph node, eye, lung, skin, brain, and pituitary gland [26]. Mammalian ASXL family proteins share highly conserved N-terminus ASXN domain, ASX homology (ASXH) domain and plant homeodomain (PHD) finger domain at $\mathrm{C}$-terminal region (Fig. 1) [27, 28]. The ASXN domain is structurally similar to forkhead-box domain and predicted to be essential for DNA-binding ability of ASXL family proteins [29, 30]. ASXH domain (also referred to as DEUBAD, deubiquitinase adaptor) is essential for interacting with BAP1 [31]. PHD domain is a histone- or DNA-binding module and is reported to recognize different subtypes of histone modifications such as unmethylated $\mathrm{H} 3 \mathrm{~K} 4(\mathrm{H} 3 \mathrm{~K} 4 \mathrm{me} 0)$ and trimethylated $\mathrm{H} 3 \mathrm{~K} 4$ (H3K4me3) [32]. What modifications does the PHD finger of ASXL1 recognize remains to be elucidated.

Human ASXL1 gene is located on chromosome 20q11 and encodes ASXL1 protein [33]. In myeloid neoplasms, most of the ASXL1 mutations are somatic ones, whereas germline mutations of ASXL1 are identified in patients with Bohring-Opitz syndrome, a developmental disorder [34]. Although ASXL2 mutations are frequently found in RUNX1-ETO fusion leukemia [35, 36], the frequency of ASXL2 mutations in other hematological neoplasms is much lower than that of $A S X L 1$ mutations [37, 38]. Unlike ASXL1
Fig. 1 The protein structure and main domains of mammalian ASXL family/Asx and BAP1/ Calypso. Known posttranslational modifications and the most frequent somatic ASXL1 mutations found in myeloid neoplasms are also shown. ASXN N-terminus ASXN domain, ASXH Asx homology domain, $P H D P H$ domain, $U C H$ Ubiquitin C-terminal hydrolase, ULD Uch37-like domain, Glu glycosylation, $U b$ ubiquitination

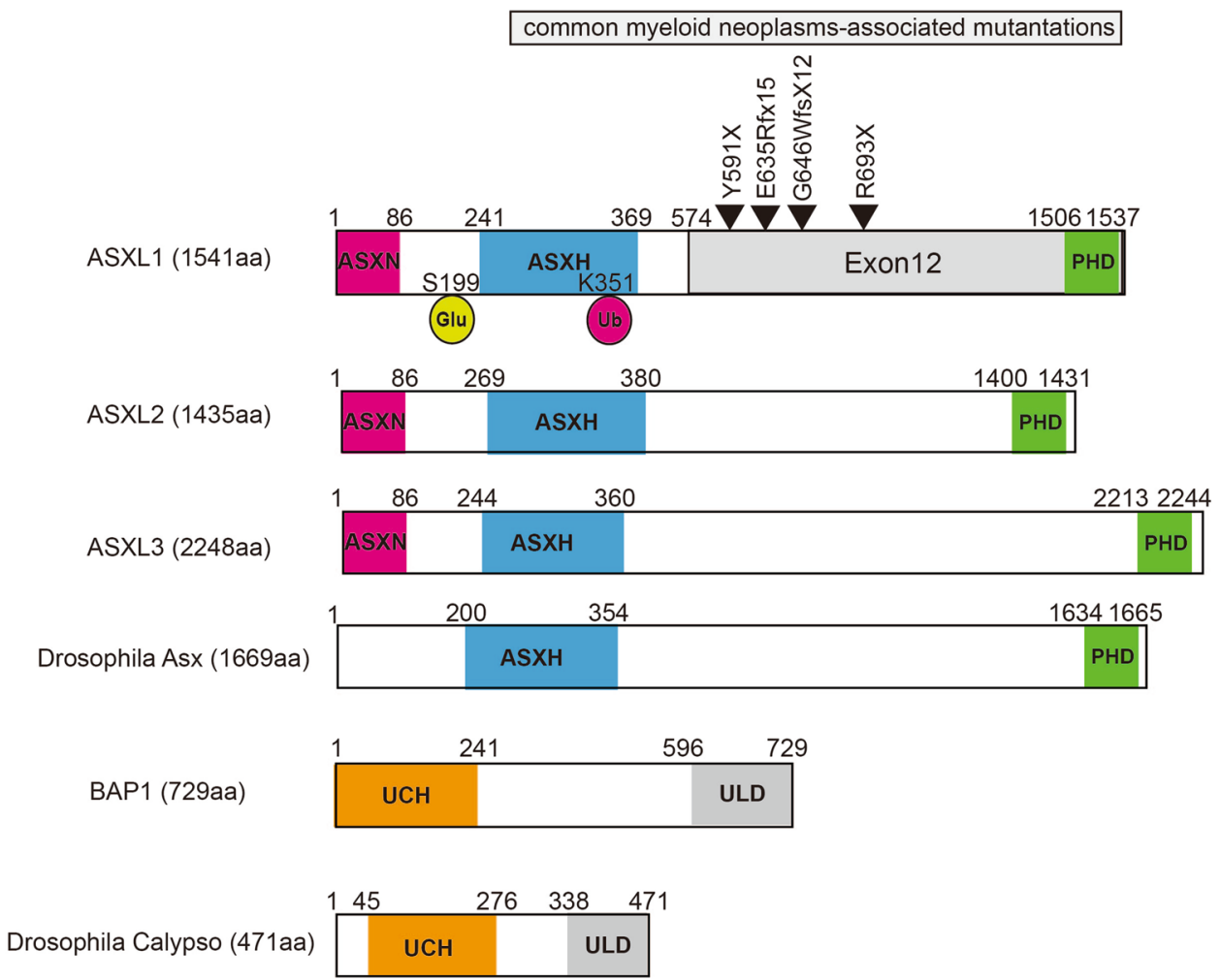


and ASXL2, ASXL3 mutations are rarely found in myeloid neoplasms [39]. This reason remains unclear, but it might be caused by the differences of DNA-binding ability of ASXN domain and the modifications recognized by PHD finger domain among ASXL1, ASXL2 and ASXL3.

\section{Altering histone modifications induced by ASXL1 loss or the mutant ASXL1 expression}

To understand the roles of wildtype ASXL1 in histone modifications, ASXL1 knockdown and Asxll knockout mouse studies have been performed. Abdel-wahab et al. demonstrated that knockdown of ASXL1 or Asxll conditional knockout mice showed substantial reduction of trimethylated H3K27 (H3K27me3) due to loss of interaction with PRC2 complex, leading to development of MDS-like disease [16, 17]. Youn et al. revealed that Asxl1 disruption in mouse embryonic fibroblasts leads to dysregulation of p16Ink4a as result of the defect in Asxl1-Ezh2 interaction [40]. These data indicated that wildtype ASXL1 is essential for maintaining the level of $\mathrm{H} 3 \mathrm{~K} 27 \mathrm{me} 3$ by interacting with PRC2 complex.

In addition, the relationship between wildtype ASXL1 and $\mathrm{H} 3 \mathrm{~K} 4 \mathrm{me} 3$ modifications has been investigated. Wang et al. also revealed that constitutive Asxll knockout mice showed global reduction not only of H3K27me3 but also of H3K4me3 [18]. Shi et al. also showed that depletion of ASXL1 resulted in impaired erythroid differentiation via reduction of the expression and $\mathrm{H} 3 \mathrm{~K} 4 \mathrm{me} 3$ of $F O X O 3$, GCN5 and MYB [41]. Inoue et al. demonstrated that ASXL1 interacts with OGT and HCFC1, and knockdown of ASXL1, OGT, HCFC1 or MLL5 attenuates myeloid differentiation and decreases global level of H3K4me3 [42]. The precise mechanisms by which loss of ASXL1 lead to a decrease in the level of $\mathrm{H} 3 \mathrm{~K} 4 \mathrm{me} 3$ remains unclear, while these data indicated that wildtype ASXL1 is also essential for maintaining the level of $\mathrm{H} 3 \mathrm{~K} 4 \mathrm{me} 3$.

However, as mentioned above, ASXL1 mutations typically occur near the last exon and its transcripts escape from nonsense-medicated decay (NMD) (Fig. 1). In patientderived cell line harboring ASXL1 mutations, the mutant ASXL1 protein was detected by Western blotting analysis and mTRAQ-based mass spectrometric analysis [19]. Therefore, it is essential to investigate how the mutant ASXL1 protein affects histone modifications and contributes to myeloid transformation. Inoue et al. demonstrated that the mutant ASXL1 binds EZH2 and overexpression of the mutant ASXL1 induced global reduction of H3K27me3 in a dominant-negative manner. The mutant ASXL1 overexpression induced derepression of miR125a and Hoxa 9 via reduction of $\mathrm{H} 3 \mathrm{~K} 27 \mathrm{me} 3$, resulting in impaired myeloid differentiation and development of MDS/AML disease in mouse BMT model [43]. Combined expression of the mutant ASXL1 and SETBP1 mutations exhibited rapid development MDS/AML in mice [44]. To investigate the role of the mutant ASXL1 at physiological expression levels, Nagase et al. generated Rosa26 locus mutant Asxll knock-in (AsxllMT KI) mice mimicking human ASXL1 E635RfsX15 mutation derived from patients with MDS/AML [45]. Asxll-MT $\mathrm{KI}$ mice displayed substantial reduction in $\mathrm{H} 3 \mathrm{~K} 4 \mathrm{me} 3$ and H2AK119ub. Unlike Asxl1 knockout mice, global level of H3K27me3 was not significantly changed in Asxll-MT KI mice. However, the level of H3K27me3 at Hoxa gene loci was decreased and the expression of Hoxa gene was also upregulated in Asxl1-MT KI mice. H3K4me3 reduction was observed especially at promoter loci of the genes associated with erythroid differentiations such as Sox6, Id3, Tjpl and Hba. Of note, overexpression of Id 3 ameliorated erythroid colonogenicity in bone marrow cells derived from Asxll-MT KI mice. Hsu et al. generated locus Asxl1 ${ }^{\text {G643fs }}$ mutant knock-in mice and reported that $A s x l 1^{\text {G643fs }}$ mutant knock-in mice alone did not develop hematological malignancies within 18 months follow-up [46]. On the contrary, Uni et al. also generated locus Asxl1 ${ }^{\text {G643fs }}$ mutant knock-in mice and reported that a part of $A s x l 1^{G 643 f s}$ mutant knock-in mice eventually developed MDS/MPN-like disease after a long latency, about 18-24 months. They demonstrated that wildtype ASXL1 but not the mutant ASXL1 can bind to BMI1, a key component of canonical PRC1 [47]. The level of $\mathrm{H} 2 \mathrm{AK} 119 \mathrm{ub}$ is decreased at p16Ink4a promoter locus, thus Ink4a expression is derepressed in Asxll ${ }^{G 643 f s}$ mutant knock-in mice. They also elucidated that $p 16$ Ink $4 a$ knockout rescues decreased HSC number and aberrant apoptosis in Asxl1 ${ }^{G 643 f s}$ mutant knock-in mice.

These findings indicated that expression of the mutant ASXL1 results in altering histone modifications and defects in hematopoiesis, leading to promoting myeloid transformation.

\section{The monoubiquitinated mutant ASXL1 forms a hyperactive complex with BAP1}

As mentioned above, BAP1 is a known interacting partner of ASXL1. BAP1 interacts with ASXL1 via DEUBAD domain [31]. BAP1 is a deubiquitinase which removes monoubiquitination of H2AK119ub. BAP1 also deubiquitinates and stabilizes non-histone targets such as IP3R3 [48], INO80 [49] and KLF5 [50]. Loss of function of BAP1 mutations are recurrently detected in melanoma [51], malignant mesothelioma [52], and kidney renal cell carcinoma [53]. Therefore, BAP1 has been considered as a tumor suppressor. In hematological neoplasms, Bap1 conditional knockout mice represented MPN/MDS-like disease due to EZH2 dysregulation 
$[54,55]$. However, a large patient data set showed BAP1 is rarely mutated in myeloid malignancies [56]. In addition, individuals with BAP1 germline mutations rarely develop myeloid malignancies [57]. There is also growing experimental evidence showing BAP1 has a tumor-promoting role cooperating with the mutant ASXL1. Balasubramani et al. showed that cancer-associated ASXL1 mutations enhanced the catalytic activity of BAP1 and profoundly reduced the global level of H2AK119ub [58]. Sahtoe et al. also demonstrated that ASXH domain is essential for recruitment of BAP1 on the chromatin [59].

Recently, we have reported that the mutant ASXL1 and BAP1 form a hyperactive complex to promote myeloid transformation (Fig. 2) [60]. As with wildtype ASXL1, the mutant ASXL1 also contains the ASXH domain (also referred to as DEUBAD), which is essential for interacting with BAP1. BAP1 expression resulted in stabilization and monoubiqutination at lysine 351 of the mutant ASXL1. The mutant ASXL1, but not wildtype ASXL1 in turn enhanced catalytic function of BAP1. Especially, the monoubiqutinated form of the mutant ASXL1 promoted nuclear localization of BAP1. As a result, the monoubiquitinated mutant ASXL1/BAP1 complex profoundly reduced the global level of H2AK119ub in hematopoietic cells. Functionally, the monoubiquitinated mutant ASXL1/ BAP1 complex inhibited the differentiation of HSPCs towards multilineage except for immature monocytes. The monoubiquitinated mutant ASXL1/BAP1 complex also promoted myeloid leukemogenesis induced by RUNX1ETO, an oncogenic fusion protein frequently coexisting with $A S X L 1$ mutations $[35,61]$. Mechanistically, this complex directly bound to promoter loci of HOXA genes and $I R F 8$, and decreases $\mathrm{H} 2 \mathrm{AK} 119$ at these loci, resulting in dysregulation of posterior HOXA genes and IRF8. HOXA gene expression is essential for leukemic transformation [62] and $I R F 8$ plays a critical role in monopoiesis [63, 64]. We also revealed that endogenous depletion of BAP1 abrogated the mutant ASXL1-induced leukemogenesis. Interestingly, endogenous BAP1 also played essential roles in promoting survival of MLL-fusion leukemia cells, which depend on HOXA gene expression. Therefore, targeting enzymatic activity of BAP1 is a promising therapeutic strategy not only for ASXL1-mutated leukemia but also for a broad range of myeloid leukemia with HOXA gene dysregulation.

As mentioned above, we demonstrated that monoubiquitination of the mutant ASXL1 is important for enhancing BAP1 activity, thereby promoting myeloid leukemogenesis. Guo et al. also revealed that reducion of Bap1 activity reduced expression of posterior Hoxa genes and attenuated leukemogenesis in the mutant ASXL1-expressing transgenic mice [65]. Recently, Daou et al. also demonstrated that monoubiquitination of ASXL2 at lysine 370 catalyzed by UBE2E family is required for deubiquitinase activity
Fig. 2 Known and proposed components associated with H2AK119ub modifications. BAP1 stabilizes and induces monoubiquitination of the mutant ASXL1. The monoubiquitinated mutant ASXL1 in turn enhances catalytic activity of BAP1. The monoubiquitinated mutant ASXL1 and BAP1 form a hyperactive PR-DUB complex, which removes ubiquitination of H2AK119 catalyzed by canonical or non-canonical PRC1 complexes. Whether UBE2Es, which monoubiquitinate ASXL2, catalyzes monoubiquitination of the mutant ASXL1 remains unknown

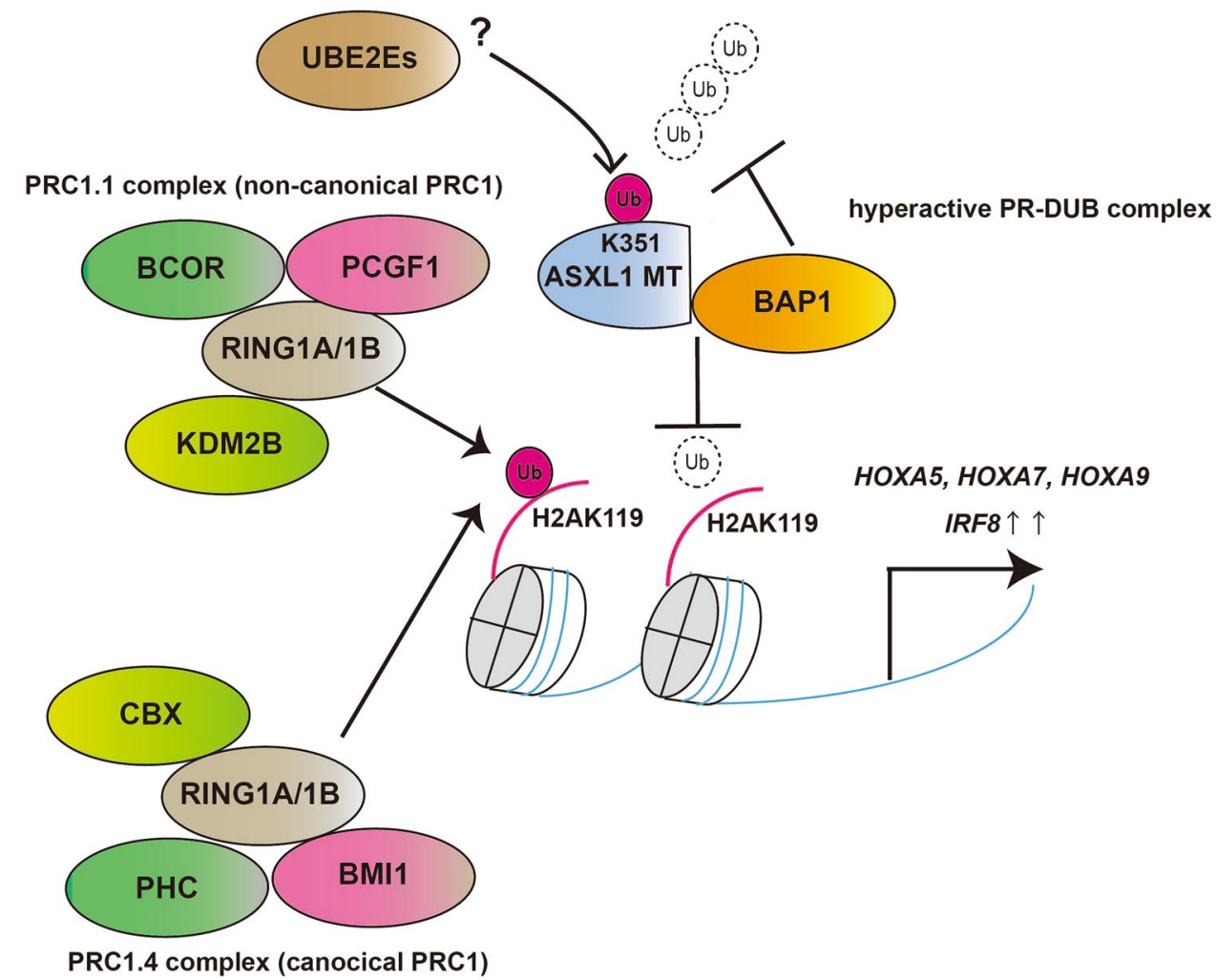


of BAP1 [66]. Further studies are required to determine whether monoubiquitination of the mutant ASXL1 is catalyzed by UBE2E family.

\section{Regulation of HOXA gene expression by H2AK119ub modification}

H2AK119ub modifications are originally identified as a repressive mark which is catalyzed by canonical PRC1 complex containing BMI1 [24, 25]. PR-DUB complex removes ubiquitin of H2AK119ub [23]. Thus, it is expected that the target genes regulated by PRC1 complex and PR-DUB complex are largely overlapped. However, recent mass spectrometry and ChIP-seq analysis revealed that the targets of PR-DUB are distinct from that of canonical PRC1 complex [67]. Actually, mammalian PRC1 family is subdivided into canonical PRC1 (cPRC1) and non-canonical PRC1(ncPRC1) [68, 69]. Gao et al. defined six major groups of PRC1 complexes, each containing a distinct PCGF subunit, a RING1A/B ubiquitin ligase, and a unique set of associated polypeptides [70].

Recently, Tara et al. demonstrated that Bcor mutation which lacks interaction with non-canonical PRC1.1 containing PCGF1 and promotes MDS/MPN development in mouse model [71]. This Bcor mutant mouse showed substantial derepression of Hoxa 7 and Hoxa 9 gene via reduction of H2AK119ub at the Hoxa7 and Hoxa9 gene loci. These data indicate that PRC1.1 complex also regulates posterior HOXA gene silencing and has tumor suppressor roles in myeloid neoplasms. A cPRC1 complex containing BMI1 (PRC1.4) is known to regulate HOXA silencing via ubiquitination of H2AK119 [72]. Therefore, the balance among PRC1.1 complex, PRC1.4 complex and PR-DUB complex will be important to maintain healthy hematopoiesis and prevent transformation via regulating HOXA gene expression (Fig. 2).

In addition, crosstalk between H2K119ub and H3K27me3 at HOXA loci remains unclear. Bapl-depleted mice showed substantial retention of H2AK119ub and increased the level of H3K27me3 [55]. On the other hand, expression of the mutant ASXL1 results in dysregulation of HOXA gene and reduction of both $\mathrm{H} 2 \mathrm{AK} 119 \mathrm{ub}$ and $\mathrm{H} 3 \mathrm{~K} 27 \mathrm{me} 3$ at $\mathrm{HOXA}$ loci $[43,45,60,65]$. In classical view, CBX recognizes H3K27me 3 catalyzed by PRC2 complex followed by recruiting of PRC1 complex to H3K27me3 and subsequent ubiquitination of H2AK119 [73]. However, a recent study showed that non-canonical PRC1.1 marks H2AK119ub at target loci and subsequently recruits PRC2 complex [69]. H2AK119ub also promotes H3K27me3 induced by Jarid2-Aebp2-containing PRC2 [74]. Further studies will shed light on precise gene regulation and histone modifications at HOXA loci.

\section{Potential therapeutic strategy for ASXL1 mutated myeloid neoplasms}

As mentioned above, BAP1 inhibition can be a promising therapeutic potential, while there are no clinically available agents inhibiting the activity of BAP1. To this date, several potential therapeutic agents including inhibitors for BRD4, OGA and HDAC have been investigated in ASXL1-mutated myeloid neoplasms (Fig. 3). Yang et al. generated C-terminal truncated mutant Asxl1 (Asxl1 ${ }^{\mathrm{Y} 588 \mathrm{X}}$ ) expressing transgenic mice and revealed that transgenic Asx11 ${ }^{\text {Y588X }}$ expression led to myeloid malignancies [75]. Mechanistically, they showed that the mutant ASXL1 protein, not wildtype ASXL1, binds to BRD4. BRD4 protein activates pTEFb complex and induces acetylation of $\mathrm{H} 3$ at lysine 122 (H3K122Ac), resulting in phosphorylation of RNA polymerase II and gene activation. In Asx11 ${ }^{\mathrm{Y} 588 \mathrm{X}}$ transgenic mice, the level of $\mathrm{H} 3 \mathrm{~K} 122 \mathrm{Ac}$ at the promoter locus of Prdml6 is increased, as a result, the expression of Prdm16 is dysregulated. Bone marrow cells from Asx11 ${ }^{\text {Y588X }}$ transgenic mice exhibited higher sensitivity toward a BRD4 inhibitor than that from normal mice. Inoue et al. demonstrated that OGT stabilized wildtype ASXL1 via glycosylation of ASXL1 at serine 199 [42]. They also revealed that PUGNAc, an OGA (O-GLcNAcase) inhibitor stabilized wildtype ASXL1 protein and restored the expression of Rara and Egrl genes via increased level of $\mathrm{H} 3 \mathrm{~K} 4 \mathrm{me} 3$ at these loci, thereby OGA inhibitor induced myeloid differentiation and showed antitumor effects on leukemia cells expressing the mutant ASXL1. Saika et al. also revealed that the mechanism by which leukemia cells combined expression with the mutant ASXL1 and mutant SETBP1 showed downregulation in TGF $\beta$ pathway was caused by reduction in acetylation of H3K14 and H4K5 at TGF $\beta$ gene loci [76]. They also demonstrated that vorinostat, a HDAC inhibitor showed substantial growth-inhibiting effects on MDS/AML cells expressing ASXL1 and/or SETBP1 mutation via restoring acetylation of H3K14 and H4K5 at TGF $\beta$ gene loci [76].

From these findings, the mutant ASXL1 altered various histone modifications and the novel agents against these alterations might be applied to clinical therapies for ASXL1-mutated myeloid malignancies.

\section{Conclusion}

Myeloid neoplasms harboring ASXL1 mutations often have poor prognosis, thereby understanding the precise mechanisms by which the mutant ASXL1 induces myeloid neoplasms is clinically needed. Recent findings from the 
Fig. 3 Overview of altered histone modifications induced by the mutant ASXL1 and potential therapeutic strategies for ASXL1-mutated myeloid neoplasms

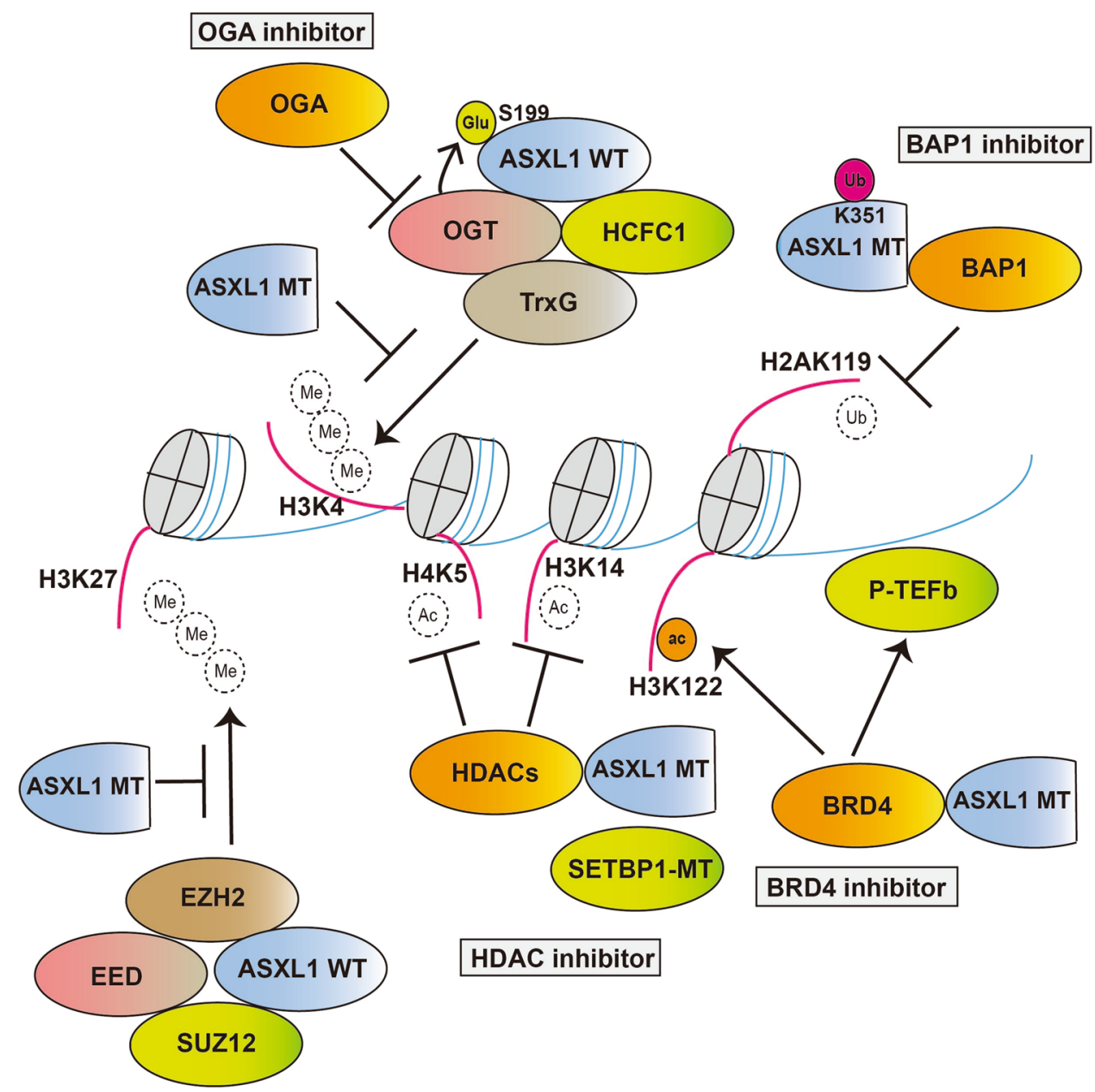

mutant ASXL1-expressing mouse studies have revealed that the mutant ASXL1 impairs hematopoiesis and promotes myeloid transformation via altering histone modifications. In addition, precise biochemical analyses have unveiled different molecular signatures between wildtype ASXL1 and the mutant ASXL1. These findings have also shed light on new potential therapeutic strategies such as BRD4 inhibition, OGA inhibition, HDAC inhibition and BAP1 inhibition. However, many unsolved riddles remain regarding functions of the mutant ASXL1 and wildtype ASXL1. For examples, the precise mechanism of how the mutant ASXL1 not wildtype ASXL1 gets stabilized in the presence of BAP1 is still unclear. In addition, hematopoietic cells expressing the mutant ASXL1 showed substantial reduction in both $\mathrm{H} 2 \mathrm{AK} 119 \mathrm{ub}$ and $\mathrm{H} 3 \mathrm{~K} 4 \mathrm{me} 3$, which play opposite roles with each other in gene regulations. Because expression of individual genes must be regulated differentially, it would be important to investigate the molecular interactions among ASXL1, MLL and SET1/COMPASS complexes as well as PRC1 and PRC2 complexes. Further studies are also required to determine which therapeutic agents are more favorable to treat with myeloid neoplasms harboring $A S X L 1$ mutations.

Acknowledgements This work was supported by a Grant-in-Aid Scientific Research B from the Ministry of Education, Culture, Sports, Science and Technology of Japan (15H04855, TK), a grant from the Tokyo Biochemical Research Foundation (TK), a grant from the Uehara Memorial Foundation (TK).

\section{Compliance with ethical standards}

Conflict of interest The authors have declared that no conflict of interest exists.

\section{References}

1. Thol F, Friesen I, Damm F, Yun H, Weissinger EM, Krauter J, et al. Prognostic significance of ASXL1 mutations in patients with myelodysplastic syndromes. J Clin Oncol. 2011;29(18):2499.

2. Bejar R, Stevenson K, Abdel-Wahab O, Galili N, Nilsson B, Garcia-Manero G, et al. Clinical effect of point mutations in myelodysplastic syndromes. N Engl J Med. 2011;364(26):2496. 
3. Schnittger S, Eder C, Jeromin S, Alpermann T, Fasan A, Grossmann $\mathrm{V}$, et al. ASXL1 exon 12 mutations are frequent in AML with intermediate risk karyotype and are independently associated with an adverse outcome. Leukemia. 2013;27(1):82.

4. Metzeler KH, Becker H, Maharry K, Radmacher MD, Kohlschmidt J, Mrozek K, et al. ASXL1 mutations identify a highrisk subgroup of older patients with primary cytogenetically normal AML within the ELN favorable genetic category. Blood. 2011;118(26):6920.

5. Pratcorona M, Abbas S, Sanders MA, Koenders JE, Kavelaars FG, Erpelinck-Verschueren CA, et al. Acquired mutations in ASXL1 in acute myeloid leukemia: prevalence and prognostic value. Haematologica. 2012;97(3):388.

6. Carbuccia N, Murati A, Trouplin V, Brecqueville M, Adelaide $\mathrm{J}$, Rey J, et al. Mutations of ASXL1 gene in myeloproliferative neoplasms. Leukemia. 2009;23(11):2183.

7. Abdel-Wahab O, Manshouri T, Patel J, Harris K, Yao J, Hedvat $\mathrm{C}$, et al. Genetic analysis of transforming events that convert chronic myeloproliferative neoplasms to leukemias. Cancer Res. 2010;70(2):447.

8. Gelsi-Boyer V, Trouplin V, Roquain J, Adelaide J, Carbuccia $\mathrm{N}$, Esterni B, et al. ASXL1 mutation is associated with poor prognosis and acute transformation in chronic myelomonocytic leukaemia. Br J Haematol. 2010;151(4):365.

9. Abdel-Wahab O, Pardanani A, Patel J, Wadleigh M, Lasho T, Heguy A, et al. Concomitant analysis of EZH2 and ASXL1 mutations in myelofibrosis, chronic myelomonocytic leukemia and blast-phase myeloproliferative neoplasms. Leukemia. 2011;25(7):1200.

10. Itzykson R, Kosmider O, Renneville A, Gelsi-Boyer V, Meggendorfer M, Morabito M, et al. Prognostic score including gene mutations in chronic myelomonocytic leukemia. J Clin Oncol. 2013;31(19):2428.

11. Patnaik MM, Padron E, LaBorde RR, Lasho TL, Finke $\mathrm{CM}$, Hanson CA, et al. Mayo prognostic model for WHOdefined chronic myelomonocytic leukemia: ASXL1 and spliceosome component mutations and outcomes. Leukemia. 2013;27(7):1504.

12. Genovese G, Kahler AK, Handsaker RE, Lindberg J, Rose SA, Bakhoum SF, et al. Clonal hematopoiesis and blood-cancer risk inferred from blood DNA sequence. N Engl J Med. 2014;371(26):2477.

13. Jaiswal S, Fontanillas P, Flannick J, Manning A, Grauman PV, Mar BG, et al. Age-related clonal hematopoiesis associated with adverse outcomes. N Engl J Med. 2014;371(26):2488.

14. Steensma DP, Bejar R, Jaiswal S, Lindsley RC, Sekeres MA, Hasserjian RP, et al. Clonal hematopoiesis of indeterminate potential and its distinction from myelodysplastic syndromes. Blood. 2015;126(1):9.

15. Jaiswal S, Natarajan P, Silver AJ, Gibson CJ, Bick AG, Shvartz $\mathrm{E}$, et al. Clonal hematopoiesis and risk of atherosclerotic cardiovascular disease. N Engl J Med. 2017;377(2):111.

16. Abdel-Wahab O, Adli M, LaFave LM, Gao J, Hricik T, Shih $\mathrm{AH}$, et al. ASXL1 mutations promote myeloid transformation through loss of PRC2-mediated gene repression. Cancer Cell. 2012;22(2):180.

17. Abdel-Wahab O, Gao J, Adli M, Dey A, Trimarchi T, Chung YR, et al. Deletion of Asxl1 results in myelodysplasia and severe developmental defects in vivo. J Exp Med. 2013;210(12):2641.

18. Wang J, Li Z, He Y, Pan F, Chen S, Rhodes S, et al. Loss of Asxl1 leads to myelodysplastic syndrome-like disease in mice. Blood. 2014;123(4):541.

19. Inoue D, Matsumoto M, Nagase R, Saika M, Fujino T, Nakayama KI, et al. Truncation mutants of ASXL1 observed in myeloid malignancies are expressed at detectable protein levels. Exp Hematol. 2016;44(3):172.
20. Katoh M. Functional proteomics of the epigenetic regulators ASXL1, ASXL2 and ASXL3: a convergence of proteomics and epigenetics for translational medicine. Exp Rev Proteom. 2015;12(3):317.

21. Sinclair DA, Milne TA, Hodgson JW, Shellard J, Salinas CA, Kyba M, et al. The Additional sex combs gene of Drosophila encodes a chromatin protein that binds to shared and unique Polycomb group sites on polytene chromosomes. Development. 1998;125(7):1207.

22. Milne TA, Sinclair DA, Brock HW. The Additional sex combs gene of Drosophila is required for activation and repression of homeotic loci, and interacts specifically with Polycomb and super sex combs. Mol Gen Genet. 1999;261(4-5):753.

23. Scheuermann JC, de Ayala Alonso AG, Oktaba K, Ly-Hartig N, McGinty RK, Fraterman S, et al. Histone H2A deubiquitinase activity of the Polycomb repressive complex PR-DUB. Nature. 2010;465(7295):243.

24. Wang H, Wang L, Erdjument-Bromage H, Vidal M, Tempst P, Jones RS, et al. Role of histone H2A ubiquitination in Polycomb silencing. Nature. 2004;431(7010):873.

25. de Napoles M, Mermoud JE, Wakao R, Tang YA, Endoh M, Appanah R, et al. Polycomb group proteins Ring1A/B link ubiquitylation of histone $\mathrm{H} 2 \mathrm{~A}$ to heritable gene silencing and $\mathrm{X}$ inactivation. Dev Cell. 2004;7(5):663.

26. Fisher CL, Randazzo F, Humphries RK, Brock HW. Characterization of Asxl1, a murine homolog of Additional sex combs, and analysis of the Asx-like gene family. Gene. 2006;369:109.

27. Katoh M, Katoh M. Identification and characterization of ASXL2 gene in silico. Int J Oncol. 2003;23(3):845.

28. Katoh M, Katoh M. Identification and characterization of ASXL3 gene in silico. Int J Oncol. 2004;24(6):1617.

29. Sanchez-Pulido L, Kong L, Ponting CP. A common ancestry for BAP1 and Uch37 regulators. Bioinformatics. 2012;28(15):1953.

30. Aravind L, Iyer LM. The HARE-HTH and associated domains: novel modules in the coordination of epigenetic DNA and protein modifications. Cell Cycle. 2012;11(1):119.

31. Daou S, Hammond-Martel I, Mashtalir N, Barbour H, Gagnon J, Iannantuono NV, et al. The BAP1/ASXL2 histone H2A deubiquitinase complex regulates cell proliferation and is disrupted in cancer. J Biol Chem. 2015;290(48):28643.

32. Sanchez R, Zhou MM. The PHD finger: a versatile epigenome reader. Trends Biochem Sci. 2011;36(7):364.

33. Fisher CL, Berger J, Randazzo F, Brock HW. A human homolog of additional sex combs, ADDITIONAL SEX COMBS-LIKE 1, maps to chromosome 20q11. Gene. 2003;306:115.

34. Hoischen A, van Bon BW, Rodriguez-Santiago B, Gilissen C, Vissers LE, de Vries P, et al. De novo nonsense mutations in ASXL1 cause Bohring-Opitz syndrome. Nat Genet. 2011;43(8):729.

35. Micol JB, Duployez N, Boissel N, Petit A, Geffroy S, Nibourel $\mathrm{O}$, et al. Frequent ASXL2 mutations in acute myeloid leukemia patients with $\mathrm{t}(8 ; 21) / \mathrm{RUNX} 1-\mathrm{RUNX} 1 \mathrm{~T} 1$ chromosomal translocations. Blood. 2014;124(9):1445.

36. Faber ZJ, Chen X, Gedman AL, Boggs K, Cheng J, Ma J, et al. The genomic landscape of core-binding factor acute myeloid leukemias. Nat Genet. 2016;48(12):1551.

37. Micol JB, Abdel-Wahab $\mathrm{O}$. The role of additional sex combs-like proteins in cancer. Cold Spring Harb Perspect Med. 2016;6(10).

38. Oak JS, Ohgami RS. Focusing on frequent ASXL1 mutations in myeloid neoplasms, and considering rarer ASXL2 and ASXL3 mutations. Curr Med Res Opin. 2017;33(4):781.

39. Duployez N, Micol JB, Boissel N, Petit A, Geffroy S, Bucci M, et al. Unlike ASXL1 and ASXL2 mutations, ASXL3 mutations are rare events in acute myeloid leukemia with $\mathrm{t}(8 ; 21)$. Leukemia Lymphoma. 2016;57(1):199.

40. Youn HS, Kim TY, Park UH, Moon ST, An SJ, Lee YK, et al. Asxl1 deficiency in embryonic fibroblasts leads to cellular 
senescence via impairment of the AKT-E2F pathway and Ezh2 inactivation. Sci Rep. 2017;7(1):5198

41. Shi H, Yamamoto S, Sheng M, Bai J, Zhang P, Chen R, et al. ASXL1 plays an important role in erythropoiesis. Sci Rep. 2016;6:28789.

42. Inoue D, Fujino T, Sheridan P, Zhang YZ, Nagase R, Horikawa $\mathrm{S}$, et al. A novel ASXL1-OGT axis plays roles in H3K4 methylation and tumor suppression in myeloid malignancies. Leukemia. 2018;32(6):1327.

43. Inoue D, Kitaura J, Togami K, Nishimura K, Enomoto Y, Uchida $\mathrm{T}$, et al. Myelodysplastic syndromes are induced by histone methylation-altering ASXL1 mutations. J Clin Invest. 2013; 123(11):4627.

44. Inoue D, Kitaura J, Matsui H, Hou HA, Chou WC, Nagamachi A, et al. SETBP1 mutations drive leukemic transformation in ASXL1-mutated MDS. Leukemia. 2015;29(4):847.

45. Nagase R, Inoue D, Pastore A, Fujino T, Hou HA, Yamasaki N, et al. Expression of mutant Asxl1 perturbs hematopoiesis and promotes susceptibility to leukemic transformation. J Exp Med. 2018;215(6):1729.

46. Hsu YC, Chiu YC, Lin CC, Kuo YY, Hou HA, Tzeng YS, et al. The distinct biological implications of Asxl1 mutation and its roles in leukemogenesis revealed by a knock-in mouse model. J Hematol Oncol. 2017;10(1):139.

47. Uni M, Masamoto Y, Sato T, Kamikubo Y, Arai S, Hara E, et al. Modeling ASXL1 mutation revealed impaired hematopoiesis caused by derepression of p16Ink4a through aberrant PRC1mediated histone modification. Leukemia. 2018. https://doi. org/10.1038/s41375-018-0198-6.

48. Bononi A, Giorgi C, Patergnani S, Larson D, Verbruggen $\mathrm{K}$, Tanji M, et al. BAP1 regulates IP3R3-mediated $\mathrm{Ca}(2+)$ flux to mitochondria suppressing cell transformation. Nature. 2017;546(7659):549.

49. Lee HS, Lee SA, Hur SK, Seo JW, Kwon J. Stabilization and targeting of INO80 to replication forks by BAP1 during normal DNA synthesis. Nat Commun. 2014;5:5128.

50. Qin J, Zhou Z, Chen W, Wang C, Zhang H, Ge G, et al. BAP1 promotes breast cancer cell proliferation and metastasis by deubiquitinating KLF5. Nat Commun. 2015;6:8471.

51. Wiesner T, Obenauf AC, Murali R, Fried I, Griewank KG, Ulz $\mathrm{P}$, et al. Germline mutations in BAP1 predispose to melanocytic tumors. Nat Genet. 2011;43(10):1018.

52. Bott M, Brevet M, Taylor BS, Shimizu S, Ito T, Wang L, et al. The nuclear deubiquitinase BAP1 is commonly inactivated by somatic mutations and 3p21.1 losses in malignant pleural mesothelioma. Nat Genet. 2011;43(7):668.

53. Pena-Llopis S, Vega-Rubin-de-Celis S, Liao A, Leng N, PaviaJimenez A, Wang S, et al. BAP1 loss defines a new class of renal cell carcinoma. Nat Genet. 2012;44(7):751.

54. Dey A, Seshasayee D, Noubade R, French DM, Liu J, Chaurushiya MS, et al. Loss of the tumor suppressor BAP1 causes myeloid transformation. Science. 2012;337(6101):1541.

55. LaFave LM, Beguelin W, Koche R, Teater M, Spitzer B, Chramiec A, et al. Loss of BAP1 function leads to EZH2-dependent transformation. Nat Med. 2015;21(11):1344.

56. Kandoth C, McLellan MD, Vandin F, Ye K, Niu B, Lu C, et al. Mutational landscape and significance across 12 major cancer types. Nature. 2013;502(7471):333.

57. Murali R, Wiesner T, Scolyer RA. Tumours associated with BAP1 mutations. Pathology. 2013;45(2):116.

58. Balasubramani A, Larjo A, Bassein JA, Chang X, Hastie RB, Togher SM, et al. Cancer-associated ASXL1 mutations may act as gain-of-function mutations of the ASXL1-BAP1 complex. Nat Commun. 2015;6:7307.

59. Sahtoe DD, van Dijk WJ, Ekkebus R, Ovaa H, Sixma TK. BAP1/ ASXL1 recruitment and activation for $\mathrm{H} 2 \mathrm{~A}$ deubiquitination. Nat Commun. 2016;7:10292.

60. Asada S, Goyama S, Inoue D, Shikata S, Takeda R, Fukushima T, et al. Mutant ASXL1 cooperates with BAP1 to promote myeloid leukaemogenesis. Nat Commun. 2018;9(1):2733.

61. Krauth MT, Eder C, Alpermann T, Bacher U, Nadarajah N, Kern $\mathrm{W}$, et al. High number of additional genetic lesions in acute myeloid leukemia with $t(8 ; 21) / R U N X 1-R U N X 1 T 1$ : frequency and impact on clinical outcome. Leukemia. 2014;28(7):1449.

62. Collins CT, Hess JL. Role of HOXA9 in leukemia: dysregulation, cofactors and essential targets. Oncogene. 2016;35(9):1090.

63. Olsson A, Venkatasubramanian M, Chaudhri VK, Aronow BJ, Salomonis N, Singh H, et al. Single-cell analysis of mixedlineage states leading to a binary cell fate choice. Nature. 2016;537(7622):698.

64. Kurotaki D, Osato N, Nishiyama A, Yamamoto M, Ban T, Sato H, et al. Essential role of the IRF8-KLF4 transcription factor cascade in murine monocyte differentiation. Blood. 2013;121(10):1839.

65. Guo Y, Yang H, Chen S, Zhang P, Li R, Nimer SD, et al. Reduced BAP1 activity prevents ASXL1 truncation-driven myeloid malignancy in vivo. Leukemia. 2018;32(8):1834.

66. Daou S, Barbour H, Ahmed O, Masclef L, Baril C, Sen Nkwe $\mathrm{N}$, et al. Monoubiquitination of ASXLs controls the deubiquitinase activity of the tumor suppressor BAP1. Nat Commun. 2018;9(1):4385.

67. Hauri S, Comoglio F, Seimiya M, Gerstung M, Glatter T, Hansen $\mathrm{K}$, et al. A high-density map for navigating the human polycomb complexome. Cell Rep. 2016;17(2):583.

68. Tavares L, Dimitrova E, Oxley D, Webster J, Poot R, Demmers $\mathrm{J}$, et al. RYBP-PRC1 complexes mediate H2A ubiquitylation at polycomb target sites independently of PRC2 and H3K27me3. Cell. 2012;148(4):664.

69. Blackledge NP, Farcas AM, Kondo T, King HW, McGouran JF, Hanssen LL, et al. Variant PRC1 complex-dependent H2A ubiquitylation drives PRC2 recruitment and polycomb domain formation. Cell. 2014;157(6):1445.

70. Gao Z, Zhang J, Bonasio R, Strino F, Sawai A, Parisi F, et al. PCGF homologs, CBX proteins, and RYBP define functionally distinct PRC1 family complexes. Mol Cell. 2012;45(3):344.

71. Tara S, Isshiki Y, Nakajima-Takagi Y, Oshima M, Aoyama K, Tanaka $\mathrm{T}$, et al. Bcor insufficiency promotes initiation and progression of myelodysplastic syndrome. Blood. 2018. https://doi. org/10.1182/blood-2018-01-827964.

72. Cao R, Tsukada Y, Zhang Y. Role of Bmi-1 and Ring1A in H2A ubiquitylation and Hox gene silencing. Mol Cell. 2005;20(6):845.

73. Cao R, Wang L, Wang H, Xia L, Erdjument-Bromage H, Tempst $\mathrm{P}$, et al. Role of histone $\mathrm{H} 3$ lysine 27 methylation in Polycombgroup silencing. Science. 2002;298(5595):1039.

74. Kalb R, Latwiel S, Baymaz HI, Jansen PW, Muller CW, Vermeulen $\mathrm{M}$, et al. Histone $\mathrm{H} 2 \mathrm{~A}$ monoubiquitination promotes histone H3 methylation in Polycomb repression. Nat Struct Mol Biol. 2014;21(6):569.

75. Yang H, Kurtenbach S, Guo Y, Lohse I, Durante MA, Li J, et al. Gain of function of ASXL1 truncating protein in the pathogenesis of myeloid malignancies. Blood. 2018;131(3):328.

76. Saika M, Inoue D, Nagase R, Sato N, Tsuchiya A, Yabushita T, et al. ASXL1 and SETBP1 mutations promote leukaemogenesis by repressing TGFbeta pathway genes through histone deacetylation. Sci Rep. 2018;8(1):15873. 